
Military Technical College, Kobry El-Kobbah, Cairo, Egypt

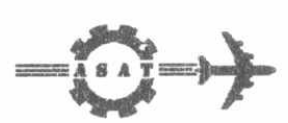

$9^{\text {th }}$ International Conference On Aerospace Sciences \& Aviation Technology

\title{
FLOATING DOCK - BREAKWATER FOR REGIONS WITH HIGH TIDAL RANGE
}

\author{
SHARAKI M.A., M.1OUSTAFA“"A.A. EL-LAKANY"* A.M.
}

\begin{abstract}
It is essential to keep the clearance between the dock surface and the ships within the allowable limits to ensure easy access and serviceability. This is hard to achieve in locations with high tidal range. Using docks that can move in the vertical direction according to variation in water level is looking attractive, especially if the dock can serve as a breakwater at the same time.

This paper introduces a simplified analytical approach to meet these requirements. The theory introduced predicts the wave height and water particle velocity in the leeward of the dock. The dimensions of the dock could be changed to obtain the suitable combination of both the wave height and water particle velocity to fulfill the functionality of the harbor and minimizing the environmental impact resulting from the existence of the dock. The model eliminates the use of the variational principle and is bas əd on mass and energy flux.
\end{abstract}

\section{KEYWORDS}

Dynamic Analysis. Added Mass, Elevated Dock, Breakwaters with gab. Environmental Effect. Transmission Coefficient Eigenfunction Expansion.

-Associate professor, Dpt. of Transpottation Engineering. Faculty of Engineening. Alexandria University. Alexandria. Egypt.

* Associate professor. Dpt. of Mathernatics \& Physics. Faculty of Engineering. Alexandria University. Alexandria. Egypt.

*-Assistant professor. Dpt. of Mathematics \& Physics. Faculty of Engineering. Alexandria University. Alexandria. Egypt. 


\section{NOMENCLATUTRE}

\begin{tabular}{|c|c|}
\hline$A_{j, 1}$ & asymmetric coefficient for potential in region I \\
\hline$A_{m i}$ & Amplitude of he incident waves mass \\
\hline B & one half width of the dock \\
\hline$c_{j, i}$ & coefficients of the hydrostatic stiffness matrix \\
\hline $\begin{array}{l}d \\
f()\end{array}$ & dock draft \\
\hline $\begin{array}{l}f() \\
F_{H}, F_{V}, F_{M}\end{array}$ & eignfunction \\
\hline$F_{H}, F_{V}, F_{M}$ & $\begin{array}{l}\text { horizontal, vertical. and moment on the fixed body } \\
\text { gab beneath the dock }\end{array}$ \\
\hline $\begin{array}{l}g \\
h\end{array}$ & gravity acceleration \\
\hline $\begin{array}{l}h \\
k_{1}\end{array}$ & water depth \\
\hline $\begin{array}{l}k_{1} \\
m_{6}\end{array}$ & wave number, eignvalue \\
\hline $\begin{array}{l}m_{6} \\
m_{1.1}\end{array}$ & mass moment about the center of rotation \\
\hline $\begin{array}{l}m_{1.1} \\
m\end{array}$ & coefficients of the mass matrix \\
\hline $\begin{array}{l}m \\
n_{1}\end{array}$ & mass of the dock \\
\hline $\begin{array}{l}n_{1} \\
R_{a}\end{array}$ & unit vector normal to the dock surface \\
\hline $\begin{array}{l}R_{a} \\
R e\end{array}$ & $\begin{array}{l}\text { asymmetric reflection coefficient of the fixed dock. } \\
\text { real part }\end{array}$ \\
\hline $\mathrm{R}_{t}$ & $\begin{array}{l}\text { real part } \\
\text { fixed reflection coefficient }\end{array}$ \\
\hline$R_{s}$ & $\begin{array}{l}\text { fixed reflection coefficient } \\
\text { symmetric reflection coefficient of the fixed dock. }\end{array}$ \\
\hline $\mathrm{S}_{j, 1 .} \mathrm{C}_{j:}$ & $\begin{array}{l}\text { symmetric reflection coefficient of the fixed dock. } \\
\text { coefficients of the potential functions }\end{array}$ \\
\hline S。 & $\begin{array}{l}\text { coefficients of the potential functions } \\
\text { the submerged (wetted) surface area }\end{array}$ \\
\hline T. & transmission coefficient of the fixed dock \\
\hline$U_{G A, 1}$ & asymmetric coefficient for potential in region II \\
\hline$U_{G S, 1}$ & symmetric coefficient for potential in region II \\
\hline $\begin{array}{l}x, y \cdot z \\
y\end{array}$ & coordinate system \\
\hline $\begin{array}{l}Y_{C G} \\
Y_{M C}\end{array}$ & elevation of mass center above the center of rotation \\
\hline o & Kronecker delta function \\
\hline$\mu_{1, j}$ & coefficients of added mass matrix \\
\hline$\stackrel{0}{P}$ & water density \\
\hline$\Phi$ & total potential \\
\hline $\begin{array}{l}\varphi \\
x_{1}\end{array}, X_{-}$ar & potential (time independent ) \\
\hline $\begin{array}{l}x_{1} \cdot x_{2}, \text { and } x \approx \\
\omega\end{array}$ & amplitudes of the motion \\
\hline$\lambda_{1}$ & $\begin{array}{l}\text { angular trequency } \\
\text { coefficients of damping matrix }\end{array}$ \\
\hline \multicolumn{2}{|l|}{ Subscripts: } \\
\hline S & sway \\
\hline h & heave \\
\hline$r$ & roll \\
\hline S & incident wave \\
\hline $\mathrm{s}$ & symmetric \\
\hline & asymmetric \\
\hline
\end{tabular}




\section{INTRODUCTION}

Mei and Black [1] used the varitional principle to solve the problem of fixed surface or submerged obstacle, Black et al [2], used the same approach to solve the same problem for a movable obstacle. Sharaki [3] introduced a simplified analytical approach to find the transmission coefficient due to the existence of elevated obstacle piercing the water surface. In the present study we extend the previous work to take into account the vertical motion of the dock.

Consider a dock with infinite length, draft $d$, width $2 B$, water depth $h$ and gab $G$ beneath it, Figure (1). The origin of the Cartesian coordinate system is located at the intersection of the still water level and the dock vertical axis of symmetry.

The horizontal axis $x$ is directed from left to right and the vertical axis $y$ points upward and the horizontal axis $z$ runs along the axis of the dock. The usual assumptions of potential and periodical flow and incompressible and inviscid fluid are used. The potential is given by

$$
\Phi(\mathrm{x}, \mathrm{y}, \mathrm{t})=\operatorname{Re}[\varphi(\mathrm{x}, \mathrm{y}) \exp (-\mathrm{i} \omega \mathrm{t})]
$$

where $\mathrm{i}=(-1)^{0.5}$, and $\mathrm{Re}$ means that only the real part is considered The potential $\varphi(x, y)$, due to any wave, incident, diffracted heave, roll or sway can be decomposed into 2 parts. A potential $\varphi_{S}$ due to symmetric flow and a potential $\varphi_{A}$ due to asymmetric flow

$$
\varphi=\varphi_{\mathrm{S}}+\varphi_{\mathrm{A}}
$$

Decomposing each potential $\varphi_{i} \varphi_{D}$. and $\varphi_{H}$ into 2 components symmetric and asymmetric potentials

$$
\varphi_{\mathrm{i}}=\varphi_{\mathrm{is}}+\varphi_{\mathrm{H}}, \varphi_{\mathrm{D}}=\varphi_{\mathrm{DS}}+\varphi_{\mathrm{DA}}, \varphi_{\mathrm{H}}=\varphi_{\mathrm{HS}}+\varphi_{\mathrm{HA}}
$$

where

$\varphi_{1} . \varphi_{C}$ and $\varphi_{H}$ potential due to the incident wave, the diffracted (scattered) wave and the heave of the dock

The domain is divided into 3 sub. domains. I. II and III. Figure (1). with the continuity equation, equation (4), and the seabed boundary conditions equation (5), satisfied in the 3 sub domains and the free surface boundary conditions. equation (6). satisfied at regions I and III,

$$
\nabla^{2} \varphi=\frac{\partial^{2} \varphi}{\partial x^{2}}+\frac{\partial^{2} \varphi}{\partial y^{2}}=0
$$




$$
\begin{aligned}
\frac{\partial \varphi}{\partial y}=0 & \text { at } y=-h \\
\frac{\partial \varphi}{\partial y}=\frac{\omega^{2}}{g} \varphi & \text { at } \quad y=0 \quad \mid x_{i}>B
\end{aligned}
$$

The components of the fluid particle velocity normal to the surface of the dock must be equal that of the dock that is.

$$
\frac{\partial \varphi}{\partial \mathbf{n}}=\mathbf{V}_{\mathbf{B}} \cdot \mathbf{n}
$$



$$
\frac{\partial \varphi}{\partial \mathbf{y}}=\mathbf{0} \quad \text { at } \mathbf{y}=-\mathbf{d}
$$

or

$$
\frac{\partial \varphi_{i}}{\partial \mathbf{n}}+\frac{\partial \varphi_{D}}{\partial \mathbf{n}}=\mathbf{0}
$$

For the vertical motion, the vertical displacement and the velocity of the dock are given by

$$
Y=\exp -i \omega t \quad Y=-i(1) \exp -i \omega t=-i(1) Y
$$

Then the boundary condition is given by

$$
\frac{\partial \Phi_{H}}{\partial \mathrm{n}}=-i \omega Y
$$




\section{THE FIXED BODY PROBLEM}

To describe the flow field for the fixed body, the potential of the incident wave, coming from left, is decomposed into two potentials, symmetric $\varphi_{i \text { is,o }}$ and asymmetric $\varphi_{i A . O}$. Each one of them contains two waves, one propagating in the positive $x$ direction and the other propagating in the negative $x$ direction. The two components of the symmetric wave are in phase, while the components of the asymmetric wave are in antiphase, Figure (1). Sharaki [3]. The following expressions describe the potential that satisfies the continuity equation, the bed and free surface boundary conditions in region I

$$
\begin{aligned}
\varphi_{1,0}=\varphi_{I S, 0}+\varphi_{I A, 0} \quad x \leq-B \\
\varphi_{I S, 0}=f_{0}(y) S_{\infty o}\left[\exp (i k(x+B))+R_{S} \exp (-i k(x+B))\right] \\
+\sum_{i=1}^{\infty} f_{i}(y) S_{0, i} \exp \left(k_{i}(x+B)\right) \\
\varphi_{I A, 0}=f_{0}(y) A_{\infty 0}\left[\exp (i k(x+B))+R_{a} \exp (-i k(x+B))\right] \\
+\sum_{i=1}^{\infty} f_{i}(y) A_{0, i} \exp \left(k_{i}(x+B)\right)
\end{aligned}
$$

with

$$
\begin{gathered}
f_{0}(y)=\frac{\cosh \left[k_{0}(y+h)\right] \sqrt{2}}{\left[h+\frac{g \sinh ^{2}\left(k_{v} h\right)}{\omega^{2}}\right]^{0.5}} \\
f_{i}(y)=\frac{\cos \left[k_{i}(y+h)\right] \sqrt{2}}{\left[h-\frac{g \sin ^{2}\left(k_{i} h\right)}{\omega^{2}}\right]^{0.5}}
\end{gathered}
$$


The functions $f_{i}(y) i=0,1,2$, are orthogonal in the interval $(-h, 0)$, that is

$$
\int_{-h}^{0} f_{i}(y) f_{j}(y) d y=\delta_{i j}
$$

Where, $\delta$ is Kronecker delta function $\left(\delta_{i j}=1: i=j, \delta_{i j}=0: i=j\right)$

and

$$
\begin{aligned}
& k_{0} \tanh \left(k_{0} h\right)=\frac{\omega^{2}}{g} \\
& k_{i} \tan \left(k_{i} h\right)=-\frac{\omega^{2}}{g}
\end{aligned}
$$

where, $k_{1}$ is the positive real root of equation (16)

The first term in $\varphi_{\text {is.o }}$ and $\varphi_{i A} .0$ corresponds to the incident wave. while the second term corresponds to the reflected wave. The terms under the summation sign give the evanescent modes, which decay rapidly with $x$

The potential in region III is given by. Sharaki [3]

$$
\varphi_{\mathrm{II}}(\mathrm{x}, \mathrm{y})=\varphi_{\mathrm{IS}}(-\mathrm{x}, \mathrm{y})-\varphi_{\mathrm{LA}}(-\mathrm{x}, \mathrm{y})
$$

The potential in region II is given by. Sharaki [3]

$$
\varphi_{\mathrm{ii.o}}=\varphi_{\mathrm{iiS.o}}+\varphi_{\mathrm{iiA.0}}=\mathrm{L}_{\mathrm{GS.o}}+\mathrm{I}_{\mathrm{GA} .0} \mathrm{x}
$$

The coefficients in equations (11-b) (11-c) and (18) are given by Sharak! [3]

$$
\begin{gathered}
\mathbf{R}_{\mathrm{S}}=\mathbf{1} \\
\mathbf{R}_{\mathrm{a}}=1+\frac{\mathrm{i} \mathbf{L}_{\mathrm{G}+\ldots} \mathbf{I}_{0}}{\mathbf{k}_{\mathrm{o}} \mathrm{S}_{\mathrm{o} a}} \\
\mathrm{~S}_{\mathrm{o}, \mathrm{i}}=0 \quad \mathrm{i}=1,2, \ldots
\end{gathered}
$$


Proceedings of the $9^{\text {th }}$ ASAT Conference, 8-10 May 2001

Paper FD -11

205

$$
\begin{aligned}
& A_{0, i}=\frac{I_{i} U_{G A, 0}}{K_{i}} \quad i=1,2, \ldots \\
& S_{00}=A_{00}=-\frac{i A_{m} g}{2 f_{0}(0)} \\
& U_{G A, 0}=-\frac{2 A_{00} I_{0}}{G B+i I_{0}^{2} / k_{0}+\sum_{i=1}^{\infty} I_{i}^{2} / k_{i}} \\
& U_{G S, 0}=2 S_{00} I_{0} / G
\end{aligned}
$$

where,

$$
I_{i}=\int_{-h}^{-d} f_{i}(y) d y
$$

The transmission and reflection coefficients for the fixed body are given by

$$
\mathbf{R}_{\mathrm{f}}=1-\frac{i \mathbf{I}_{\mathrm{o}}^{2}}{\mathbf{k}_{\mathrm{o}} B G+i I_{\mathrm{o}}^{2}+\mathbf{k}_{\mathrm{o}} \sum_{\mathrm{i}=1}^{\infty} \mathrm{I}_{\mathrm{i}}^{2} / \mathbf{k}_{\mathrm{i}}}
$$

and

$$
T_{f}=\frac{i I_{0}^{2}}{k_{0} B G+i I_{0}^{2}+k_{0} \sum_{i=1}^{\infty} I_{i}^{2} / k_{i}}
$$

The forces and moment on the body due the hydrodynamic pressure, are given by Sharaki[3].

$$
\begin{aligned}
F_{H} & =\left[\int_{-d}^{d} P(-B, y)-P(B, y) d y\right] \\
& =2 i \omega \rho\left[\overline{\mathbf{I}}_{0} A_{o}\left(1+R_{a}\right)+\sum_{i=1}^{\infty} \overline{\mathbf{I}}_{i} A_{o, i}\right] \exp (-i \omega t)
\end{aligned}
$$


or

$$
F_{H}=4 i \omega \rho A_{0} 。\left[\bar{I}_{c}-T_{r}\left(\bar{I}_{0}-i \frac{k_{0}}{\bar{I}_{0}} \sum_{i=1}^{\infty} \bar{I}_{i} I_{i} / k_{i}\right)\right] \exp (-i \omega t)
$$

for the horizontal force, and

$$
F_{M}=2 i \omega\left[\begin{array}{l}
A_{0 \circ}\left(1+R_{A}\right)\left(d \bar{I}_{0}+\hat{I}_{0}\right) \\
+\sum_{i=1}^{\infty} A_{0, i}\left(d \bar{I}_{i}+\hat{I}_{i}\right)+\frac{1}{3} U_{G A, 0} B^{3}
\end{array}\right] \exp (-i \omega t)
$$

or

$$
F_{M}=4 i \omega \rho A_{0.0}\left[\hat{I}_{0}+T_{f}\left(\hat{I}_{0}-\frac{i_{k_{0}}}{I_{0}}\left\{\sum_{i=1}^{\infty} \hat{I}_{i} I_{i} / k_{i}+B^{3} / 3\right\}\right)\right] \exp (-i \omega t)
$$

for the moment, and

$$
F_{V}=i \omega \rho \int_{-B}^{B} U_{G S} d x=2 i \omega \rho B U_{G S .0} \exp (-i \omega t)
$$

for the vertical force, where

$$
\begin{aligned}
& \hat{\mathbf{I}}_{\mathrm{i}}=\int_{-\mathrm{d}}^{0} \mathrm{y} \mathrm{f}_{\mathrm{i}}(\mathrm{y}) \mathrm{dy} \\
& \overline{\mathbf{I}}_{\mathrm{i}}=\int_{-\mathrm{d}}^{0} \mathbf{f}_{\mathrm{i}}(\mathbf{y}) d
\end{aligned}
$$

\section{THE MOVING BODY}

To find the potential due to heave sway or roll motion of unit amplitude the potential in region I is given by 


$$
\begin{aligned}
& \varphi_{1, j}=\varphi_{I S, J}+\varphi_{I A, j} \\
& =f_{0}(y)\left[S_{j, 0} \exp \left(-i k_{0}(x+B)\right)\right]+\sum_{i=1}^{\infty} f_{i}(y) S_{j, i} \exp \left(k_{i}(x+B)\right)+ \\
& f_{0}(y)\left[A_{j, 0} \exp \left(-i k_{0}(x+B)\right)+\right]+\sum_{i=1}^{\infty} f_{i}(y) A_{j, i} \exp \left(k_{i}(x+B)\right) \\
& y \leq B
\end{aligned}
$$

The subscript $j$ is 1 for the sway, 2 for the heave and 6 for the roll. The potential for region II is selected according to the motion considered. The first term in equation (34) represent wave propagating to the left. The terms under the summation sign correspond to the evanescent modes that decay with the horizontal distance For the heave motion only the symmetric part is considered, while for the sway and roll motions only the asymmetric part is considered. The flow in region III is still given by equation (17).

\section{The Heave Motion}

In this paper we consider only the heave (vertical) motion, then equation (34) takes the form

$$
\begin{aligned}
\varphi_{1,2} & =\varphi_{1 S, 2} \\
& =f_{0}(y)\left[S_{2,0} \exp \left(-i k_{0}(x+B)\right)\right]+\sum_{i=1}^{\infty} f_{i}(y) S_{2, i} \exp \left(k_{i}(x+B)\right):: x \leq B
\end{aligned}
$$

For region II, a potential that satisfies the distribution of the velocity given in figure (1) is given by

$$
\varphi_{11.2}=C_{2.1}\left(y^{2}+2 h y-x^{2}\right)+C_{2.2}
$$

with

$$
\frac{\hat{c} \varphi_{\mathrm{a} .2}}{\partial \mathbf{x}}=-2 \mathrm{C}_{2,1} \mathbf{x} \cdot \text { and } \frac{\hat{c} \varphi_{\mathrm{a} . z}}{\hat{\partial} \mathrm{y}}=\mathrm{C}_{2,1}(2 \mathrm{y}+\mathbf{2 h})
$$

The first equation, the horizontal velocity, is a linear function increasing linearly The first equation, the his. This is in agreement with the type of flow expected to occur due to the vertical movement of the dock. The second equation satisfies the bottom boundary condition. To find the constant $C_{2,1}$ we use the continuity 
equation. Assume that, the vertical displacement and velocity of the pontoon are given by

$$
Y=\exp -i \omega t \quad \dot{Y}=-i \omega \exp -i \omega t=-i \omega Y
$$

The change in volume in time dt, must equal the discharge along the two vertical lines, separating region II from regions I and III, this gives:

$$
C_{2,1}=-\frac{i \omega}{2 G}
$$

And the potential is given by

$$
\varphi_{\mathbb{D} .2}=-\frac{i \omega}{2 G}\left(y^{2}+2 h y-x^{2}\right)+C_{2.2}
$$

This gives, $\ddot{Y}=-i \omega$ at $y=-d$ which is equal to the vertical velocity of the pontoon.

The potential at region 1 is given by equation (35). To find the coefficients $C_{2}$, we equate the velocity at $x=-B$ on both sides of the vertical line separating regions I and II.

$$
\begin{aligned}
S_{2, o} & =\frac{\omega B I_{0}}{K_{0} G} \\
S_{2, j} & =-\frac{i \omega B I_{j}}{G K_{j}} \quad j=1,2 \ldots
\end{aligned}
$$

$C_{2.2}$ is found by equating the total pressure at both sides of the gab at $x=-B$ This gives

$$
C_{2.2}=\frac{\omega B I_{0}^{2}}{k G^{2}}-\frac{i \omega B}{G^{2}} \sum_{j=1}^{\infty} \frac{I_{j}^{2}}{k_{j}}+\frac{i \omega}{2 G}\left(\frac{G^{2}}{3}-h^{2}-B^{2}\right)
$$

\section{THE EXITING FORCES, ADDED MASSES AND DAMPING}

The forces and moments on the body due to the fluid pressure acting on the submerged part of the body consist of two parts. The first part is due to the hydrostatic pressure and is a function of the instantaneous position of the body. The second is due to the hydrodynamic pressure.

The general equation of motion, in matrix form, is given by 


$$
\sum_{i=1,2,6} \mathbf{m}_{j i} \frac{\partial^{2} \alpha_{i}}{\partial \mathbf{t}^{2}}=-\sum_{i=1.2,6} c_{j i} \alpha_{i}+\rho \int_{S_{0}} \frac{\partial \varphi}{\partial t} n_{j} d S \quad j=1,2,6
$$

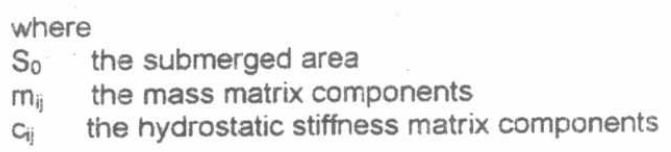

the subscript j,i are for 1 for the sway, 2 for the heave and 6 for the roll, respectively

The mass matrix components include the body mass, the mass moment of inertia and the mass products of inertia. The hydrostatic stiffness matrix components are defined in terms of the body waterline the location of the center of gravity and center of buoyancy. Expressions for $m_{i j}$ and $c_{i j}$ are given by Newman, 1977. When external forces, such as mooring forces, act on the body, the equations of motion must be modified to take care of such forces. The same argument applies equally when there is damping in the system.

The hydrodynamic forces are given by the last term of equation (40). These are usually divided into two components, the exiting force $F_{e}$ due to the incident and scatted (diffracted) potentials $\phi_{i}$ and $\phi_{\mathrm{s}}$ ( (the exiting potential $\phi_{\mathrm{e}}=\phi_{1}+\phi_{\mathrm{s}}$ ) and the added mass and damping Forces $F_{f}$ due to the forced potentials $\phi_{1}, \phi_{2}$ and $\phi_{6}$. $(\phi)$ Once more, we are interested only in the heave motion.

\section{The Exiting Forces and Moments}

The horizontal and vertical forces and moment experienced by the fixed body due to the incident and scattering waves (the exiting potential $\phi_{e}$ ), are given by integrating the pressure. given by the last term of equation (40), along the wetted surface. using $n_{1}, n_{2}$ and $n_{6}$ respectively.

The horizontal force, vertical force and moment are given by. Sharaki [3]

$$
\begin{aligned}
& F_{e, 1}=4 i \omega \rho A_{0,0}\left[\vec{I}_{0}+T_{i}\left(\bar{I}_{o}-\frac{i k_{u}}{I_{0}} \sum_{i=1}^{\infty} \bar{I}_{i} I_{i} / k_{i}\right)\right] \exp (-i \omega t) \\
& F_{e, 2}=i \omega \rho \int_{-B}^{B} U_{G A} d x=2 i \omega \rho B U_{G A, 0} \exp (-i \omega t) \\
& F_{e, 6}=4 i \omega \rho A_{0.0}\left[\hat{\mathbf{I}}+T_{i}\left(\hat{I}_{0}-\frac{i_{k_{o}}}{I_{0}}\left\{\sum_{i=1}^{\infty} \hat{\mathbf{I}}_{i} I_{i} / k_{i}+B^{3} / 3\right\}\right)\right] \exp (-i \omega t)
\end{aligned}
$$


where

$\bar{I}_{1}=\int_{-b}^{i} f_{i}(y) d y$

and

$$
\hat{I}_{1}=\int_{-d}^{n} y f(y) d y
$$

It should be mentioned that $F_{e, 1}, F_{e, 2}, F_{e, 6}$ could be calculated in terms of the incident potential and the forced potential without using the scattering potential. Using Green's theory, we can do this by introducing expressions that represent the exiting forces directly in terms of the incident and forced potentials. Those expressions are called Haskind relations, Mei[4], and may be given in the form

$$
F_{e, j}=\rho \int_{S_{0}}\left[\varphi_{i} \frac{\partial \varphi_{j}}{\partial n}-\varphi_{j} \frac{\partial \varphi_{1}}{\partial n}\right] d s \quad j=1,2,6
$$

\section{The Added Mass and Damping}

The forces and moment components $F_{i j} j=1,2,6$ resulting from the forced potential, $\phi_{t}$ are obtained in a manner exactly similar to that for the forces and moment due to the exiting potential $\phi_{\mathrm{e}}$. They are given by:

$$
F_{r, j}=\rho \int_{S} \frac{\partial \varphi_{r}}{\partial t} n_{j} d S=-i \omega \rho \sum_{i=1,2.6} \int_{S}\left(\varphi_{r, i} n_{j} d S\right) \alpha_{i}
$$

Those equations can be decomposed into components in phase with the velocity and the acceleration of each mode as follows. Sarpkaya and Isaacson $[5]$

$$
\begin{aligned}
\mathbf{F}_{\mathrm{r}, \mathrm{j}} & =-\sum_{\mathrm{i}=1.2,6}\left(\mu_{\mathrm{ji}} \frac{\partial^{2} \boldsymbol{\alpha}_{\mathrm{i}}}{\partial \mathbf{t}^{2}}+\lambda_{\mathrm{ji}} \frac{\partial \boldsymbol{\alpha}_{\mathrm{i}}}{\partial \mathrm{t}}\right) \quad \text { for } \mathrm{j}=1,2,6 \\
& =-\sum_{\mathrm{i}=1.2 .6}\left(-\omega^{2} \mu_{\mathrm{ji}}-\mathrm{i} \omega \lambda_{\mathrm{ji}}\right) \exp -\mathrm{i} \omega \mathrm{t}
\end{aligned}
$$

$H_{i i}$ and $\lambda_{i i}$ are called the added mass and damping coefficients respectively They are named so because they play the rule of mass and damping in the equation of motion. Those coefficients are real. Equating the right hand sides of equations (47) and (48), then $\mu_{j}$ and $\lambda_{j i}$ are given by. Sarpkaya and Isaacson [5]

$$
\mu_{\mathrm{ji}}=\frac{p}{\omega} \int_{\mathrm{S}} \operatorname{Im}\left[\varphi_{\mathrm{i}}\right] \mathbf{n}_{\mathrm{j}} \mathrm{dS}=\frac{\mathrm{i} \rho}{\omega^{2}} \int_{\mathrm{S}} \operatorname{Im}\left[\varphi_{\mathrm{i}}\right] \frac{\partial \varphi_{\mathrm{j}}}{\partial \mathrm{n}} \mathrm{dS}
$$




$$
\lambda_{\mathrm{ji}}=-\rho \int_{\mathrm{S}} \operatorname{Re}\left[\varphi_{\mathrm{i}}\right] \mathrm{n}_{\mathrm{j}} \mathrm{dS}=--\frac{\mathrm{i} \rho}{\omega} \int_{\mathrm{S}} \operatorname{Re}\left[\varphi_{\mathrm{i}}\right] \frac{\partial \varphi_{\mathrm{j}}}{\partial \mathrm{n}} \mathrm{dS}
$$

$\mu_{i j}$ and $\lambda_{j i}$ are obtained by integrating the pressure along the submerged surface of the pontoon in the heave, roll and sway motions and separating the real and imaginary parts. Using equation (41), (42), (43), and (48), then the equation of motion (40) takes the form

$$
\sum_{i}\left(m_{j i}+\mu_{j i}\right) \frac{\partial^{2} \alpha_{i}}{\partial t^{2}}+\sum_{i} i_{j i} \frac{\partial \alpha_{i}}{\partial t}+\sum_{i} \quad c_{j i} \alpha_{i}=F, \quad j=1,2,6
$$

Since the heave motion is uncoupled with both the roll and sway motions, then

$$
\begin{aligned}
& \mu_{21}=\mu_{12}=\mu_{62}=\mu_{26}=0 \\
& \lambda_{21}=\lambda_{12}=\lambda_{62}=\lambda_{26}=0
\end{aligned}
$$

According to equations (49), and (50), one can write

$$
\begin{aligned}
& \mu_{22}=\frac{\rho}{\omega} \operatorname{Im}\left(\mathrm{P}_{\mathrm{r}, 22}\right) \\
& \lambda_{22}=-\rho \operatorname{Re}\left(\mathrm{P}_{\mathrm{r}, 22}\right)
\end{aligned}
$$

Where $P_{r, 22}$ is given by

$$
\begin{aligned}
P_{r .22} & =\int_{-B}^{+B} \varphi_{H .2} n_{2} d x \quad \text { at }(y=-d) \\
& =-\frac{i \omega B}{G}\left[d^{2}-2 d h-B^{2} / 3\right]+2 B C_{2.2}
\end{aligned}
$$

It should be noted that. only the potential in region $\mid \mathrm{I}$ is considered. The added mass and damping due to roll and sway may be found in a similar manner assuming that the appropriate potential for roll and sway motions can be determined. However. in this paper we are interested only on the heave motions. 


\section{THE EQUATION OF MOTION}

The equation of motions of the dock, in matrix form is given, by, [5]

$$
\left[\begin{array}{ccc}
\mathbf{M}_{22} & 0 & 0 \\
0 & \mathbf{M}_{11} & \mathbf{M}_{61} \\
0 & \mathbf{M}_{16} & \mathbf{M}_{66}
\end{array}\right]\left[\begin{array}{l}
\chi_{2} \\
\chi_{1} \\
\chi_{6}
\end{array}\right]=\left[\begin{array}{l}
\mathbf{F}_{e .2} \\
\mathbf{F}_{e .1} \\
\mathbf{F}_{e .6}
\end{array}\right]
$$

Where

$$
\begin{aligned}
& M_{22}=\left(-\omega^{2}\left(\mathbf{m}+\mu_{22}\right)-i \omega \lambda_{22}+2 \rho g B+S_{2}\right) \\
& M_{66}=\left(-\omega^{2}\left(m_{6}+\mu_{66}\right)-i \omega \lambda_{66}+m g \mathbf{I}_{M C}+S_{6}\right) \\
& M_{11}=\left(-\omega^{2}\left(m+\mu_{11}\right)-i \omega \lambda_{11}+S_{1}\right) \\
& M_{61}=\left(-\omega^{2} \mu_{61}-i \omega \lambda_{61}\right) \\
& M_{16}=\left(-\omega^{2} \mu_{16}-i \omega \lambda_{16}+\omega^{2} m Y_{C G}\right)
\end{aligned}
$$

The third term in equation (56-a) represent the buoyancy, while the last term $S_{2}$ represents the vertical stiffness of the pontoon. $S_{1}$ and $S_{6}$ represent the horizontal and rotational stiffness. respectively. $Y_{C G}$ is the elevation of the mass center above the center of rotation. $m$ is the mass of the body. $Y_{M C}$ is the metacenter height. $m_{6}$ is the mass moment about the center of rotation. The third term in equation (56-b) represent the hydrostatic restoring moment resulting from the rotation of the pontoon. $X_{1}, X_{2}$ and $X_{5}$ are the amplitudes of the sway. heave and roll motions respectively. Since we are interested only in the heave motion. then the equation of motion. equation (55) reduces to

$$
\mathbf{M}_{2: 2} \chi_{2}=\mathbf{F}_{\mathrm{e}: 2}
$$

\section{THE EFFICIENCY OF THE PONTOON}

The transmission coefficient of the dock may be defined as the ratio between the surface elevation at both sides of the breakwater. The surface elevation is given by

$$
\boldsymbol{\eta}=-\frac{1}{\mathrm{~g}} \frac{\hat{\partial} \Phi}{\hat{c} \mathbf{t}} \quad \text { at } \mathbf{y}=\mathbf{0}
$$


Using equations (17) and (58), and noting that the roll and sway motions are asymmetric while the heave motion is symmetric. Also noting that the amplitude asymmetric while breakwater is $A_{\mathrm{m}}$. The surface elevation in the lee of the dock is given as the sum of the elevations due to the incident wave, the heave, the sway and the roll. Dividing the resulting surface elevation by the surface elevation in the seaside of the dock gives the transmission coefficient

$$
T=\frac{1}{A_{m i}}\left[T_{f} A_{m i}+\frac{i \omega}{g}\left(\varphi_{1,2} \chi_{2}-\varphi_{1,1} \chi_{1}-\varphi_{1,6} \chi_{6}\right)\right] \quad \text { at } y=0
$$

It should be noted that the minus signs in the previous equation result from equation (17) and the fact that both the roll and sway motions are asymmetric. One may drop the evanescent modes, since they decay rapidly with distance from the dock. Also, since the factor $\exp \left(i k_{0}(x+B)\right)$ is common to all terms, then it could be factored out. The transmission coefficient takes the form

$$
\mathbf{T}=\mathbf{T}_{\mathrm{f}}+\frac{\mathrm{i} \omega}{\mathbf{g A}_{\mathrm{mi}}}\left(\chi_{2} \mathbf{S}_{2,0}-\chi_{1} \mathbf{A}_{1,0}-\chi_{6} \mathbf{A}_{6,0}\right)
$$

However, since this paper considers only the heave motion, then the transition coefficient is given by setting $X_{1}$ and $X_{6}$ equals zeros. This leads to

$$
T=T_{f}+\frac{i \omega}{g_{A_{m i}}}\left(\chi_{2} S_{2,0}\right)
$$

The phase difference, $\theta_{i}$, between the incident wave and the transmitted wave and the phase difference, $\theta_{h}$, between the incident waves and the waves created due to the heave of the dock are given by

$$
\begin{aligned}
& \theta_{i}=\tan ^{-1}\left(\operatorname{Im}\left(T_{f}\right) / \operatorname{Re}\left(T_{f}\right)\right) \\
& \theta_{h}=\tan ^{-1}\left(\operatorname{Im}\left(i \omega \chi_{2} S_{2.0}\right) / \operatorname{Re}\left(i \omega \chi_{2} S_{2,0}\right)\right)
\end{aligned}
$$

Hence the phase lag. $\theta_{L}$, between the transmitted waves and the heave waves is given by $\theta_{L}=\theta_{i}-\theta_{h}$. If the dimensions of the dock and the vertical stiffiness are selected such that $\theta_{L}$ is greater than $\pi / 2$ then the efficiency of the dock, as a breakwater. will exceeds that of the fixed dock.

If the variation in the water level due to tidal effect is $h$, from the optimum position, then, from vertical equilibrium, the dock clearance change $V$, is given by

$$
V=\alpha d_{e} h /\left(1+d_{e} \alpha\right)
$$


where, $d_{e}$ is the draft in the optimum position and $a$ is the ratio between the vertical stiffness and the dry weight of the dock.

\section{CASE STUDY}

Consider a dock located in a region with tidal range $1.00 \mathrm{~m}$. The LWL is: $3.00 \mathrm{~m}$. The HWL is $4.00 \mathrm{~m}$. The dock width, $2 \mathrm{~B}$, is $12.00 \mathrm{~m}$. The draft is $2.5 \mathrm{~m}$. When the water level is 3.5 , which is considered the optimum position. The transmission coefficients, the amplitudes of the vertical displacement of the dock due to the passage of the wave and the change of the clearance between the dock and the mean water level due to the tidal effect are calculated for water depths 3.00. 3.50 and $4.00 \mathrm{~m}$, respectively. The previous quantities are calculated for vertical stiffness $0.0 .5 W$ and $W$ respectively, where $W$ is the weight of the dock Table (1) shows the draft $d$, gab $G$, and change in clearance $V$ for the cases considered

Figures (2-a) through (2-f) show the transmission coefficients and the amplitudes of the vertical motions due to the waves passing the dock for the cases mentioned before.

For the cases studied, the figures show that for smaller depth, increasing the stiffness of the vertical mooring has minimal effect on the transmission coefficients. However. when the depth increases due to the tide. docks with higher mooring stiffness produce less transmission coefficients. In all cases increasing the vertical stiffness reduces the amplitude of the motion due to the wave passing. In contrary. this increases the clearance between the dock and the water surface, as can be seen from Tabie (1). Figure (3) shows how the transmission coefficient is affected by changing the vertical stiffness while keeping all other factors constant

\section{CONCLUSION}

This paper use truncated eignfunction expansion to describe the flow field due to both diffraction and radiation. resulting from heave motion for a box-like structure. The method can be extended to cover the cases of a mooring structure once the potential due to unit amplitude of roll and sway motions are known. For regions with high tidal range and moderate waves the theory proposes constructing a dock-breakwater structure to function as both a dock and a breakwater. This is important for regions such as the Red Sea where it is essential not to endanger the cora! reef while at the same time having recreational locations. In addition to predict the transmission coefficient, the method predicts the amplitude of the dock motion due to the heave. With suitabie selection of the dimension of the structure and the properties of the vertical spring the dock can satisfies all the requirements of creating calm water and comfortable docking operation 


\section{REFERANCES}

1- Mei, C. C. and Black, J. L. , Scattering of Surface Waves by Obstacles in Waters of Finite Depth. J of Fluid Mechanics, Vol. 38, part 3, pp. 499511, 1969

2- Black, J. L., Mei C. C. and Bray M. C. G., Radiation of Scattering of Water waves by Rigid Bodies, J of Fluid Mechanics Vol. 46, part 1 pp. 151-164, 1971

3- Sharaki, M.A, Docks and Breakwaters with Minimum Environmental Impact for Small Boat Harbors and Marinas, accepted for publication, Alexandria Engineering Journal.

4-Mei, C. C., The Applied Dynamic of Ocean Surface Waves, World Science Publication Co. 1992.

5- Sarpkaya, T and Isaacson. M Mechanics of Wave forces on Offshore Structures, Van Noostrand Reinhold Company 1981. 
Table 1. draft d, gab $G$ and change in clearance $V$.

\begin{tabular}{|c|c|c|c|c|}
\hline $\begin{array}{l}\text { Water } \\
\text { depth }\end{array}$ & & ST2 $=00$. & $\mathrm{ST} 2=0.5 \mathrm{~W}$ & ST2 $=W$ \\
\hline \multirow{3}{*}{$3.0 \mathrm{~m}$} & $d$ & 2.50 & 2.22 & 2.15 \\
\hline & $\mathrm{V}$ & 0.00 & 0.28 & 0.36 \\
\hline & $G$ & 050 & 0.78 & 0.85 \\
\hline \multirow{3}{*}{$3.5 \mathrm{~m}$} & d & 2.50 & 2.50 & 2.50 \\
\hline & $\mathrm{V}$ & 0.00 & 0.00 & 0.00 \\
\hline & G & 1.00 & 1.00 & 1.00 \\
\hline \multirow{3}{*}{$4.0 \mathrm{~m}$} & $d$ & 2.50 & 2.78 & 2.86 \\
\hline & V & 0.00 & 0.28 & 0.36 \\
\hline & G & 1.50 & 1.22 & 1.14 \\
\hline
\end{tabular}

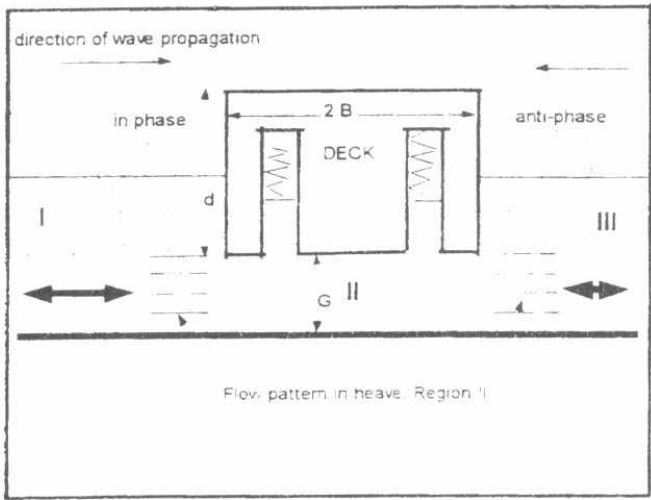

Figure (1). Definition Sketch of the Dock and wave decomposition 

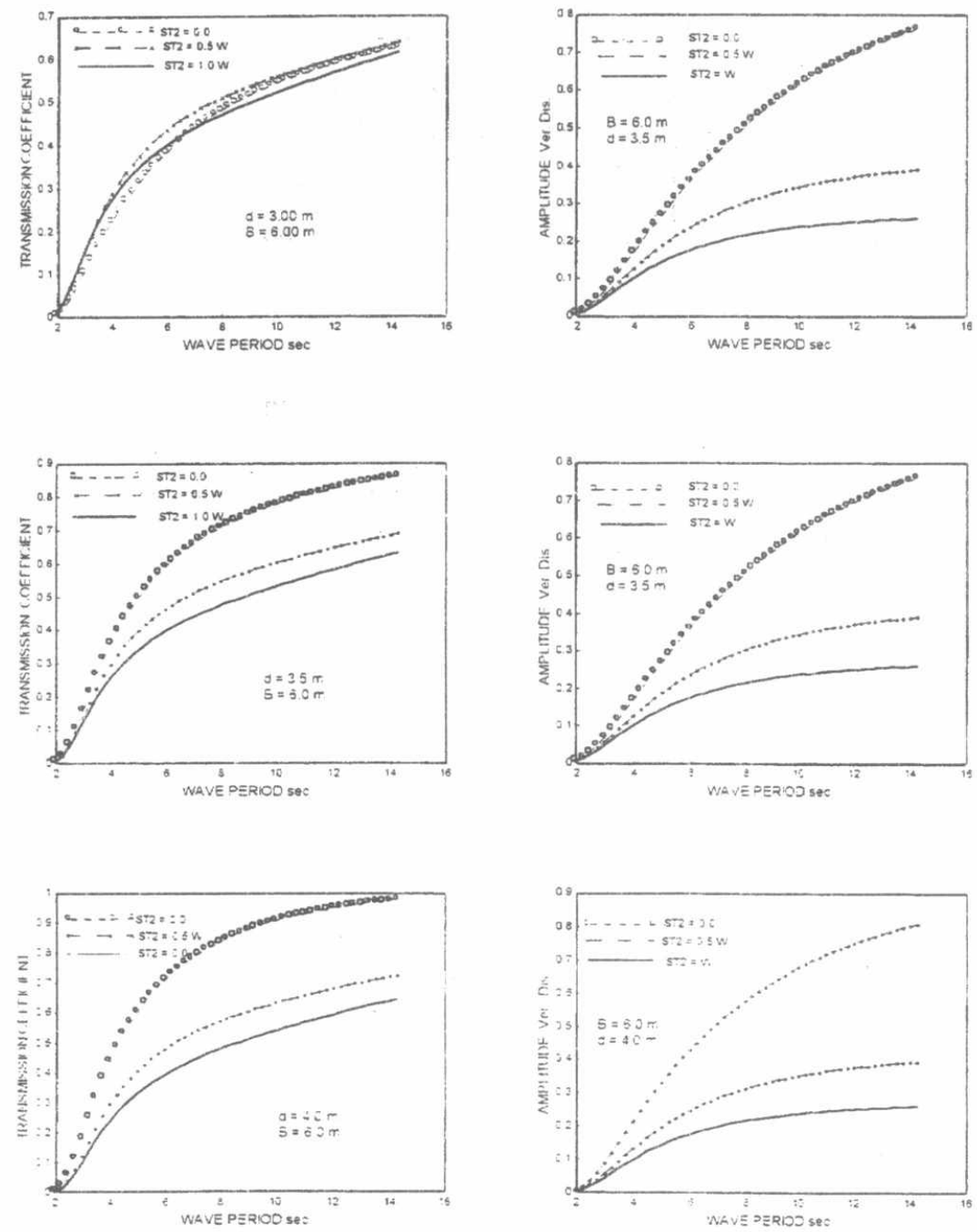

Figure (2-a, 2-f) Transmission coefficient and amplitude of the vertical motion 


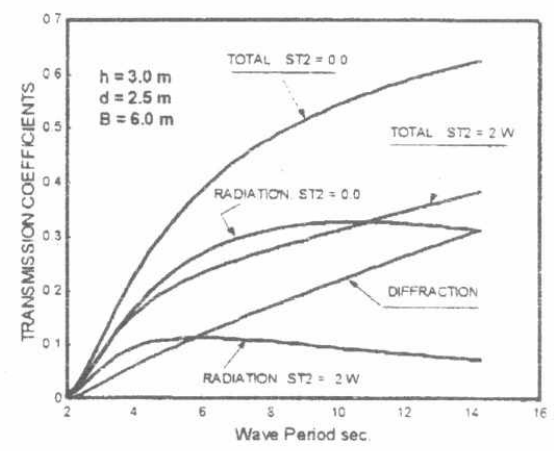

Figure 3. Variation in the transmission coefficient with the mooring stiffness 